\title{
Towards Verifiable Adaptive Flight Control for Safety Critical Applications
}

\author{
Mac Schwager* and Anuradha M. Annaswamy ${ }^{\dagger}$ \\ Massachusetts Institute of Technology, Cambridge, MA, 02139 \\ Eugene Lavretsky \\ The Boeing Company, Huntington Beach, CA, 92647
}

\begin{abstract}
One of the main obstacles to the implementation of adaptive controllers for safety critical applications is the absence of analytically justified Verification and Validation (V\&V) techniques for such systems. This paper seeks to provide the beginnings of a theoretically motivated $V \& V$ technique for adaptive controllers in the context of controlling uncertain flight vehicle dynamics. A set of tools for characterizing the transient properties of direct adaptive systems is developed using a combination of Lyapunov theory, asymptotic analysis, and linear systems theory. A Lyapunov approach is used to prove stability and global properties. Asymptotic analysis allows for the behavior of the nonlinear adaptive system to be practically characterized by a Reduced Linear Asymptotic System (RLAS). Techniques used in linear systems theory then can be applied straightforwardly to the RLAS. The tools are demonstrated on a simulation of the short period mode in aircraft dynamics and on a full nonlinear six degree of freedom aircraft simulation.
\end{abstract}

\section{Introduction}

$\mathrm{T}$ HE application of adaptive control to aircraft promises benefits in safety and robustness and is considered to be one of the main enabling technologies for Unmanned Air Vehicles (UAV's). Early attempts at adaptive flight control used controllers with unproven stability properties, sometimes with disastrous consequences; for example the fatal crash of the NASA X-15 in November, 1967. As a result, much of the theoretical work up to the present time has been rightly focused on stability of adaptive architectures. Currently, there exists an assortment of stable adaptive control strategies, as well as techniques for preserving stability in the presence of unknown, bounded disturbances. ${ }^{1,2}$ In addition, recent military interest in UAV's has caused a surge of adaptive flight control research. UAV's provide an exciting test ground for experimental control techniques. It is reasonable to expect that the viability of adaptive flight control systems must be proven in unmanned vehicles prior to their implementation in manned aircraft, where safety is a much more critical consideration. For this reason, the development of stable and robust adaptive flight control systems for UAV's is a crucial gateway to the broader acceptance of adaptive control strategies for other safety critical applications.

Three common adaptive architectures have been investigated in conjunction with aircraft control. Perhaps the most promising architecture is direct adaptive control, in which control parameters are adapted based on some performance error. Direct adaptive strategies were explored in Refs. 3-6. Another common adaptive control architecture, indirect adaptive control, uses a controller derived from a plant model, while the model is continuously updated using system identification techniques. A notable application of an indirect adaptive flight controller is treated in Ref. 7 using a multiple model approach. Finally, neural network based flight controllers have also been a popular topic of research. ${ }^{8,9}$ Such controllers use one or more networks of basis functions, which are adapted online using learning algorithms. Each of these control techniques has been investigated in myriad embodiments and variations. In addition, methods such as Training Signal Hedging (TSH) have been developed to overcome the realworld problem of saturating actuators. ${ }^{10-12}$ In Ref. 13, TSH was used to develop a direct-adaptive controller for simultaneous control allocation and reconfiguration.

\footnotetext{
* Graduate Student, Mechanical Engineering Department, 77 Massachusetts Ave. Rm. 1-107.

${ }^{\dagger}$ Principal Research Scientist, Mechanical Engineering Department, 77 Massachusetts Ave. Rm. 3-461B.

* Technical Fellow, Phantom Works, The Boeing Company, Huntington Beach, CA 92647
} 
Based on this substantial body of research, it can be argued that the study of adaptive flight control systems has reached sufficient maturity to warrant research into more practical problems of integrating adaptive controllers with aircraft systems. Currently, the chief practical obstacle to transitioning adaptive flight controllers into aerospace applications is an inability to analytically assert that the closed-loop system will have adequate stability / robustness margins, acceptable transient behavior, and will provide the necessary disturbance rejection properties. This is not a trivial task because the dynamics of an adaptive system in closed loop are nonlinear. For example, there is currently no simple analytical technique to determine whether or not a control signal produced by a given adaptive controller will exceed the bandwidth of control actuators. Similarly, there is no simple technique to determine whether or not the response of a given adaptive control system will produce frequencies that may interact with, for instance, unmodelled structural modes. Such concerns can be grouped under the umbrella of Verification and Validation $(\mathrm{V} \& \mathrm{~V})$ and are obviously of paramount importance in application to aircraft and other safety critical systems. However these concerns have received curiously little attention in the adaptive control literature. Researchers have generally relied on extensive simulation and trial and error to produce adaptive control systems with suitable transient properties.

The V\&V techniques that are currently in use for modern aircraft systems ${ }^{14-17}$ are unsuitable for adaptive flight control systems because they rest on the assumption that the control system is linear (at least locally). The need for completely new V\&V techniques is expanded on in Refs. 18 and 19, and some necessary features of a successful $\mathrm{V} \& \mathrm{~V}$ procedure are laid out in Ref. 20. Some specific techniques have been proposed for neural network based controllers. For example, the method in Ref. 21 relies on bounding neural network outputs using Lipschitz conditions imposed on the chosen set of basis functions, and a second method employs Support Vector Machines (SVM) to determine if a neural network will produce an output that is out of specification. ${ }^{22}$ These methods are specific to neural network based adaptive control systems, and it is difficult to envision their use in an industry setting due to their complicated and theoretical nature.

The contribution of this paper is to introduce a set of tools based on Lyapunov theory, asymptotic analysis, and linear systems theory for analyzing the transient behavior and disturbance rejection properties of adaptive systems. At the same time, the tools provide practical guidelines for tuning adaptive controllers to satisfy predetermined performance criteria. The focus of this paper is limited to the simplest embodiment of a direct adaptive controller: Model Reference Adaptive Control (MRAC) using state feedback for a single input plant. In section II, the problem is introduced and its scope is discussed. In section III, the analysis tools are derived and their relevance to the problem is proven. Section IV applies the tools to the design and analysis of an adaptive flight controller for a linear short period dynamics of a fixed-wing aircraft with various uncertainties. Finally, in section V, the tools are applied to a full, nonlinear, six degree of freedom (6-DoF) aircraft simulation, and their viability is demonstrated as a practical V\&V procedure for safety critical adaptive systems. Conclusions and directions for future research are given in section VI.

\section{Problem Statement}

\section{A. Model Development}

The problem under consideration is the control of an uncertain, states accessible, nonlinear plant of the form

$$
\dot{X}=f_{p}(X, U)
$$

where $X \in \mathfrak{R}^{n}$, and $U \in \mathfrak{R}$. For the purposes of control, the nonlinear plant is approximated by a schedule of Linear Time-Invariant (LTI) systems of the form

$$
\dot{x}=A_{p} x+b_{p} \delta_{a d}+d_{p}
$$

$$
\text { where } x=X-X_{0} \text {, and } \delta_{a d}=U-U_{0} \text {, }
$$

and where, $A_{p} \in \mathfrak{R}^{n \times n}, b_{p} \in \mathfrak{R}^{n}$, and $d_{p} \in \mathfrak{R}^{n}$ are unknown. $X_{0}$ is a desired equilibrium state and $U_{0}$ is an unknown constant "trim" input that maintains the plant at $X_{0}$ in the absence of the disturbance $d_{p}$. Specifically, $X_{0}$ 
and $U_{0}$ satisfy the relation $b_{p} U_{0}=-A_{p} X_{0}$. It is desired that the plant follow a known reference model, which itself is nonlinear and of the form

$$
\dot{X}_{m}=f_{m}\left(X_{m}, U_{m}\right)
$$

where $X_{m} \in \mathfrak{R}^{n}$, and $U_{m} \in \mathfrak{R}$ is a bounded input from a pilot or a guidance/navigation system. Moreover, the reference model is also expressed as a schedule of known LTI systems with a known interpolation algorithm. Each LTI reference model can be written as:

$$
\begin{gathered}
\dot{x}_{m}=A_{m} x_{m}+b_{m} \delta_{c}, \\
\text { where } x_{m}=X_{m}-X_{0} \text {, and } \delta_{c}=U_{m}-U_{m 0},
\end{gathered}
$$

It is assumed that $A_{m}$ is Hurwitz, $X_{0}$ is the same equilibrium state used in Eq. (1), and $U_{m 0}$ is the necessary trim input for the reference model, so that $b_{m} U_{m 0}=-A_{m} X_{0}$.

In this work, each LTI plant-reference model pair (Eqs. (1) and (2)) will be treated separately, as is common in flight dynamics. It is assumed that the above scheduled representation of a nonlinear system is adequate for the problem at hand and questions pertaining to inaccuracies of this representation will not be considered in this paper. The representation described above is typical of flight vehicle dynamics. Note that Eq. (1) may include physical dynamics as well as feedback dynamics imposed by the presence of a nominal controller, and the state $x$ may include controller states, such as integrators, as well as other physical parameters.

\section{B. Direct Adaptive Controller}

We would like to design a control input $\delta_{a d}\left(x, x_{m}, \delta_{c}\right)$ such that

$$
\lim _{t \rightarrow \infty}\left(x-x_{m}\right)=0
$$

Let the input, $\delta_{a d}$, be given by the control law

$$
\begin{gathered}
\delta_{a d}=\theta^{T} \omega, \\
\text { where } \omega=\left[\begin{array}{lll}
x & \delta_{c} & 1
\end{array}\right]^{T} \text {, and } \theta=\left[\begin{array}{lll}
\theta_{x}^{T} & \theta_{\delta} & \theta_{d}
\end{array}\right]^{T},
\end{gathered}
$$

and $\theta_{x} \in \mathfrak{R}^{n}, \theta_{\delta} \in \mathfrak{R}$, and $\theta_{d} \in \mathfrak{R}$ are control gains. The control gains are adjusted according to the adaptation law

$$
\dot{\theta}=-\Gamma \omega b_{m}^{T} P e
$$

where $e=x-x_{m}$ is the system tracking error, $P$ is the unique symmetric positive definite solution of the algebraic Lyapunov equation $A_{m}^{T} P+P A_{m}=-Q$, with $Q>0$. Also, in (6), $\Gamma>0$ is a positive definite symmetric matrix of adaptation rates. Assuming that there exist ideal gains $\theta_{x}^{*}, \theta_{\delta}^{*}>0$, and $\theta_{d}^{*}$ such that

$$
b_{p} \theta_{x}^{* T}=A_{m}-A_{p}, b_{p} \theta_{\delta}^{*}=b_{m}, \text { and } b_{p} \theta_{d}^{*}=-d \text {, }
$$

the controller in Eq. (4) with the control law in Eq. (6) can be shown to achieve tracking as specified in Eq. (3). Equations (7) are known as the model matching conditions. The control gains can then be redefined in terms of the ideal gains and gain errors as 


$$
\widetilde{\theta}_{x}=\theta_{x}-\theta_{x}^{*}, \widetilde{\theta}_{\delta}=\theta_{\delta}-\theta_{\delta}^{*} \text {, and } \widetilde{\theta}_{d}=\theta_{d}-\theta_{d}^{*},
$$

Substituting Eqns. (1), (2), (4), (6), (7), and (8), and recalling that $e=x-x_{m}$ gives

$$
\left[\begin{array}{c}
\dot{e} \\
\dot{\tilde{\theta}}
\end{array}\right]=\left[\begin{array}{cc}
A_{m} & \lambda_{\delta} b_{m} \omega^{T} \\
-\Gamma \omega b_{m}^{T} P & 0
\end{array}\right]\left[\begin{array}{l}
e \\
\widetilde{\theta}
\end{array}\right],
$$

where, $\lambda_{\delta}=\frac{1}{\theta_{\delta}^{*}}$, and $\widetilde{\theta}=\left[\begin{array}{ccc}\widetilde{\theta}_{x}^{T} & \widetilde{\theta}_{\delta} & \widetilde{\theta}_{d}\end{array}\right]^{T}$. Equation (9) represents the error dynamics of the closed loop adaptive system. Notice that the error dynamics are nonlinear and time varying due to the presence of the linear regressor vector $\omega$. The problem considered in this paper is how to design adaptation gains $\Gamma$ and $Q$ in Eq. (9) to produce an adaptive response for a given specification, and, once designed, how to verify that the response is indeed within the given specification.

\section{Adaptive Control Design/Analysis Tools}

\section{A. Tool Development}

The tools are developed in two theorems which are then proven. The first theorem states the well known properties of MRAC systems based on Lyapunov analysis. The second theorem introduces the Linear Asymptotic System (LAS), which emerges from an asymptotic analysis of Eq. (9). The LAS is then simplified to give the Reduced Linear Asymptotic System (RLAS).

\section{Theorem 1}

The error dynamics in Eq. (9) have the following properties:

i) The plant state $x$ is bounded.

ii) The controller gains $\theta$ are bounded

iii) $\lim _{t \rightarrow \infty} e=0$.

\section{Proof of Theorem 1}

Consider the Lyapunov function candidate $V=e^{T} P e+\widetilde{\theta}^{T} \lambda_{\delta} \Gamma^{-1} \widetilde{\theta}$. Taking time derivatives along the system trajectories gives $\dot{V}=-e^{T} Q e \leq 0$. This implies that $V$ is bounded, and hence $e$ and $\widetilde{\theta}$ are bounded. Since $A_{m}$ is stable and $\delta_{c}$ is bounded, $x_{m}$ is bounded. This, in turn, implies that $x$ and $\theta$ are bounded and i) and ii) are proved. Now, $x$ bounded and $\delta_{c}$ bounded imply that $\omega$ is bounded; and $A_{m}$ stable, $e$ bounded, and $\widetilde{\theta}$ bounded imply that $\dot{e}$ is bounded. This implies that $\ddot{V}$ is bounded. Therefore, by Barbalat's lemma, $\lim _{t \rightarrow \infty} \dot{V}=0$, which directly implies iii).

\section{Definition 1}

A dynamics, $\dot{z}_{1}=f\left(z_{1}\right)$, is said to converge to another dynamics, $\dot{z}_{2}=g\left(z_{2}\right)$ if, given an $\varepsilon, \exists T$ such that $\forall$ initial condition $z_{1}\left(t_{0}\right)$ and $z_{2}\left(t_{0}\right)$,

$$
\left\|z_{1}(t)-z_{2}(t)\right\| \leq \varepsilon \quad \forall t \geq t_{0}+T
$$

\section{Theorem 2}

For a constant input, $\delta_{c}$, the error dynamics in Eq. (9) converge to the dynamics

$$
\left[\begin{array}{c}
\dot{e}_{a} \\
\dot{\tilde{\theta}}_{a}
\end{array}\right]=\left[\begin{array}{cc}
A_{m}+\lambda_{\delta} b_{m} \theta_{x c}^{T} & \lambda_{\delta} b_{m} \omega_{m_{c}}^{T} \\
-\Gamma \omega_{m_{c}} b_{m}^{T} P & 0
\end{array}\right]\left[\begin{array}{c}
e_{a} \\
\widetilde{\theta}_{a}
\end{array}\right],
$$


where $\omega_{m_{c}} \in \mathfrak{R}^{n+2}$ is a known constant, and $\lambda_{\delta} \in \mathfrak{R}$ and $\theta_{x_{c}} \in \mathfrak{R}^{n}$ are unknown bounded constants.

\section{Proof of Theorem 2}

The linear regressor, $\omega$, can be written

$$
\begin{gathered}
\omega=\omega_{e}+\omega_{m}, \\
\text { where } \omega_{e}=\left[\begin{array}{lll}
e^{T} & 0 & 0
\end{array}\right]^{T} \text { and } \omega_{m}=\left[\begin{array}{lll}
x_{m}^{T} & \delta_{c} & 1
\end{array}\right]^{T} .
\end{gathered}
$$

With $\delta_{c}$ constant and $A_{m}$ stable, $\omega_{m}$ converges exponentially to a constant $\omega_{m c}$, where

$$
\omega_{m_{c}}=\left[\begin{array}{lll}
x_{m_{c}}^{T} & \delta_{c} & 1
\end{array}\right]^{T} \text {, and } x_{m_{c}}=-A_{m}^{-1} b_{m} \delta_{c}
$$

Therefore the error dynamics in (9) converge exponentially to a new error dynamics which can be expressed as

$$
\left[\begin{array}{c}
\dot{e}_{t} \\
\dot{\tilde{\theta}}_{t}
\end{array}\right]=\left[\begin{array}{cc}
A_{m} & \lambda_{\delta} b_{m} \omega_{m_{c}}^{T} \\
-\Gamma \omega_{m_{c}} b_{m}^{T} P & 0
\end{array}\right]\left[\begin{array}{c}
e_{t} \\
\widetilde{\theta}_{t}
\end{array}\right]+\left[\begin{array}{c}
\lambda_{\delta} b_{m} \omega_{e_{t}}^{T} \widetilde{\theta}_{t} \\
-\Gamma \omega_{e_{t}} b_{m}^{T} P e_{t}
\end{array}\right],
$$

where $\omega_{e t}^{T}=\left[\begin{array}{lll}e_{t}^{T} & 0 & 0\end{array}\right]^{T}$. Notice that Eq. (11) is time-invariant and nonlinear. It has been shown that ${ }^{23}$ for a constant input, $\delta_{c}$, the parameter error $\widetilde{\theta}_{t}$ converges to an unknown constant vector $\theta_{c}=\left[\begin{array}{lll}\theta_{x_{c}}^{T} & \theta_{\delta_{c}} & \theta_{d_{c}}\end{array}\right]^{T}$. Using this fact and Theorem 1, the dynamics in (11) can be expanded in a Taylor series about the equilibrium point $e_{t}=0$ and $\widetilde{\theta}_{t}=\theta_{c}$ to give

$$
\left[\begin{array}{c}
\dot{e}_{a} \\
\dot{\tilde{\theta}}_{a}
\end{array}\right]=\left[\begin{array}{cc}
A_{m}+\lambda_{\delta} b_{m} \theta_{x_{c}}^{T} & \lambda_{\delta} b_{m} \omega_{m_{c}}^{T} \\
-\Gamma \omega_{m_{c}} b_{m}^{T} P & 0
\end{array}\right]\left[\begin{array}{c}
e_{a} \\
\widetilde{\theta}_{a}
\end{array}\right]+\left[\begin{array}{c}
f\left(e_{a}, \widetilde{\theta}_{a}\right) \\
g\left(e_{a}, \widetilde{\theta}_{a}\right)
\end{array}\right],
$$

where $f$ and $g$ contain only second order terms. Using Definition 1 , Theorem 2 follows directly.

The properties of chief interest for $\mathrm{V} \& \mathrm{~V}$ are the asymptotic state error dynamics, $e_{a}$, and the input dynamics, $\delta_{a d}$. Define the asymptotic input error dynamics to be

$$
\widetilde{\delta}_{a}=\omega_{m_{c}}^{T} \widetilde{\theta}_{a}
$$

Substituting (13) into (10) and simplifying gives

$$
\left[\begin{array}{c}
\dot{e}_{a} \\
\dot{\tilde{\delta}}_{a}
\end{array}\right]=\left[\begin{array}{cc}
A_{m}+\lambda_{\delta} b_{m} \theta_{x c}^{T} & \lambda_{\delta} b_{m} \\
-\gamma b_{m}^{T} P & 0
\end{array}\right]\left[\begin{array}{c}
e_{a} \\
\widetilde{\delta}_{a}
\end{array}\right],
$$

where $\gamma$ is defined to be the known scalar $\omega_{m_{c}}^{T} \Gamma \omega_{m_{c}}$. Equation (14) is denoted as the Reduced Linear Asymptotic System (RLAS). Notice that the LAS (Eq. (10)) has $2 n+2$ eigenvalues, $n+1$ of which are stable and the remaining $n+1$ are identically zero. The RLAS has $n+1$ stable eigenvalues, which are equal to the $n+1$ stable eigenvalues of the LAS. The RLAS is a simple, linear, compact approximation to the dynamics of the closed loop adaptive system and is the main tool used in this paper to predict the oscillatory behavior of the adaptive system for design or verification purposes. 


\section{B. RLAS Design Procedure}

The optimal selection of $\Gamma$ and $Q$ in Eq. (9) for a given set of performance metrics is the eventual goal of our research. The RLAS is useful toward this end because $\Gamma$ and $Q$ can be designed for the RLAS using linear design techniques to meet the given performance metrics. The same $\Gamma$ and $Q$ can then be applied to the adaptive system, which is proven to converge to the RLAS, and therefore, will satisfy the performance metrics as well, after the transient delay. In this paper we take a first step toward this goal and find $\Gamma$ and $Q$ by a combination of analysis and numerical simulations.

In order to find the optimal $\Gamma$ and $Q$, the unknown quantities $\lambda_{\delta}$ and $\theta_{x c}$ in Eq. (14) must be estimated. $\lambda_{\delta}$, the control effectiveness uncertainty, was chosen as an example of an extraordinary failure, though in practice, expected bounds on $\lambda_{\delta}$ would have to be determined based on the specifics of the aircraft and the mission. The asymptotic gain error, $\theta_{x c}$, was estimated in an iterative process in the following way. The unknown value $\theta_{x c}$ was set to zero in the RLAS (Eq. (14)) and suitable values of $\Gamma$ and $Q$ were determined using the root locus method. The trajectory of the adaptive dynamics (Eq. (9)) was then found through numerical simulation using these values of $\Gamma$ and $Q$, and a new value of $\theta_{x c}$ was determined. This process was repeated until a value of $\theta_{x c}$ was converged upon. In practice, only one or two iterations were required since it was found that the RLAS dynamics were typically not sensitive to variations in $\theta_{x c}$. This observation can be verified with a root locus of the RLAS with respect to each of the elements of $\theta_{x c}$. Currently we are investigating methods for analytically estimating $\theta_{x c}$, or bounding its effect on the RLAS, to avoid this iterative procedure.

We now discuss how $\Gamma$ and $Q$ are determined for a given value of $\theta_{x c}$. It is known in a qualitative sense that increasing $\Gamma$ and $Q$ will cause more vigorous oscillation and less overshoot in an adaptive system. The proposed RLAS design approach allows these intuitions to be formalized. In the RLAS the pilot/navigation system input, $\delta_{c}$, and the adaptive gain $\Gamma$ appear as a single scalar, $\gamma$. A root locus of Eq. (14) with respect to $\gamma$ can be used to verify the intuition that increasing $\Gamma$ increases response frequency. Likewise, the RLAS gives the additional insight that the response frequency is roughly proportional to the square of the input signal $\delta_{c}$. Thus if one wishes to limit the frequency of the adaptive response, one must have some control, or at least some known bounds, on the input signal. The effects of the elements of the vector $b_{m}^{T} P$ can be determined similarly, noting that increasing these elements leads to a more oscillatory response. Therefore, $\gamma$ and $b_{m}^{T} P$ can be designed for the RLAS using the common arsenal of linear design techniques, such as the root locus method, Bode plots, or Nyquist plots. These can then be used to back calculate $\Gamma$ and $Q$ straightforwardly, taking care that both $P$ and $Q$ in the linear Lyapunov equation are positive definite. For the studies in this paper $\Gamma$ and $Q$ were determined using the root-locus method to obtain suitable eigenvalues for the RLAS. In particular, these values were chosen so that the oscillatory mode of the RLAS corresponded to a given design specification, while all $1^{\text {st }}$ order modes were sufficiently fast not to inhibit response time.

\section{Design Verification}

The RLAS can further be used to verify the adaptive design by comparing its trajectory to that of the adaptive system. For this procedure, we explicitly simulate the response of the RLAS and the adaptive system under some known failure (or uncertainty) to ascertain how close their responses are and how quickly they converge. The RLAS was formulated as previously (Eq. (14)) using the same values of $\lambda_{\delta}$ and $\theta_{x c}$. To give a meaningful comparison with the trajectory of the adaptive system, the RLAS trajectory must be added to the reference model trajectory to obtain a corresponding linear "state" trajectory. For this purpose, an appropriate set of initial conditions $e_{a}\left(t_{0}\right)$ and $\widetilde{\delta}_{a}\left(t_{0}\right)$ for the RLAS must be calculated from the corresponding conditions of the adaptive system at $t_{0}$. The initial error, $e_{a}\left(t_{0}\right)$ is easily found from the initial states of the adaptive system and reference model as $e_{a}\left(t_{0}\right)=x\left(t_{0}\right)-x_{m}\left(t_{0}\right)$, while the initial input error $\widetilde{\delta}_{a}\left(t_{0}\right)$ is found from $\widetilde{\delta}_{a}\left(t_{0}\right)=\omega_{m_{c}}\left(\theta\left(t_{0}\right)-\theta^{*}\right)$. Comparison of the trajectories can then be used to infer whether the adaptive system satisfies the design criteria. In the following sections we will apply such a design/verification methodology to design an adaptive controller with suitable transient properties for a linear model of the aircraft short period mode and for a full nonlinear 6-DOF aircraft model.

\section{Application to Aircraft Short Period Dynamics}

In this section, the usefulness of the RLAS approach is demonstrated using linear uncertain short period aircraft dynamics with a state feedback nominal controller. The adaptive controller is applied to the closed loop nominal system in an architecture that will be referred to as adaptive augmentation. The design and verification capabilities of the RLAS are then demonstrated in a simulation environment. 


\section{A. Model and Nominal Controller Development}

From Ref. (24), short period dynamics of a fixed-wing aircraft with zero bank angle can be expressed as:

$$
\left[\begin{array}{c}
\dot{\alpha} \\
\dot{q}
\end{array}\right]=\left[\begin{array}{cc}
-L_{\alpha} & -L_{q} \\
\lambda_{\alpha} M_{\alpha} & \lambda_{q} M_{q}
\end{array}\right]\left[\begin{array}{c}
\alpha \\
q
\end{array}\right]+\lambda_{\delta}\left[\begin{array}{c}
0 \\
M_{\delta}
\end{array}\right]\left(\delta+d_{t r m}\right) .
$$

In Eq. (15), $\alpha$ is the aircraft angle of attack (AOA), $q$ is the body pitch rate. The scalars $\lambda_{\delta}>0, \lambda_{\alpha}$, and $\lambda_{q}$, represent uncertainties in the parameter values, and $d_{t r m}$ denotes an unknown trim input component. In addition, it is assumed that the aircraft state vector, $x=[\alpha q]^{T}$, is available on-line for control purposes. The rest of the parameters represent the so-called aircraft stability and control derivatives. The values of the stability and control derivatives used in this example are

$$
L_{\alpha}=0.6582, L_{q}=-0.9705, M_{\alpha}=-3.3105, M_{q}=-1.4741 \text {, and } M_{\delta}=-3.6764 \text {. }
$$

They were found from a linearization of a nonlinear aircraft model, (see section V for details). The dynamics in Eq. (15) can be expressed compactly as

$$
\dot{x}=A x+\lambda_{\delta} b\left(\delta+d_{t r m}\right) .
$$

Likewise, let the dynamics without uncertainty (i.e., Eq. (15) where $\lambda_{\alpha}=\lambda_{q}=\lambda_{\delta}=1$, and $d_{t r m}=0$ ), be denoted

$$
\dot{x}_{s p}=A_{s p} x_{s p}+b_{s p} \delta
$$

A nominal controller is designed assuming no uncertainty as in Eq. (17) and applied to the uncertain dynamics in Eq. (15). The Linear Quadratic (LQ) optimal control design technique ${ }^{25}$ is straightforwardly applied to the dynamics in Eq. (17). A state-feedback controller architecture is used for the nominal controller so that

$$
\delta_{n o m}=k_{x}^{T} x+k_{\delta} \delta_{c},
$$

where $k_{x}=\left[k_{\alpha} k_{q}\right]^{\mathrm{T}}$. The feedback gain, $k_{x}$, is found by minimizing the cost function

$$
J=\frac{1}{2} \int_{0}^{\infty}\left(x^{T}\left(Q_{J}+k_{x}^{T} R k_{x}\right) x d t\right.
$$

subject to the dynamics in Eq. (17). A suitable closed loop response is found with $Q_{J}=\operatorname{diag}([21])$ and $R=1$ to give the feedback gains $k_{x}=\left[\begin{array}{lll}-0.2816-0.7434\end{array}\right]^{T}$. The feed forward gain, $k_{\delta}$, is designed to produce angle of attack following so that $k_{\delta}=1 / g_{\alpha}$, where $\left[\begin{array}{ll}g_{\alpha} & g_{q}\end{array}\right]^{T}=-\left[A_{s p}+b_{s p} k_{x}\right]^{-1} b_{s p}$ is the steady state gain of Eq. (17) with the feedback component $k_{x}$. Then the closed loop dynamics of Eq. (17) with control law (18) can be written

$$
\dot{x}_{m}=A_{m} x_{m}+b_{m} \delta_{c}
$$

where $A_{m}=\left(A_{s p}+b_{s p} k_{x}\right)$ and $b_{m}=b_{s p} k_{\delta}$. Defining the input to the actual dynamics (Eq. (16)) to be $\delta=\delta_{\text {nom }}+\delta_{a d}$, the actual closed dynamics becomes:

$$
\dot{x}=\left(A+\lambda_{\delta} b k_{x}\right) x+\lambda_{\delta} b k_{\delta} \delta_{c}+\lambda_{\delta} b\left(\delta_{a d}+d_{t r m}\right),
$$

\section{B. Adaptive Augmentation and RLAS}


Assume that we desire for the uncertain closed loop system (Eq. 21)) to follow the closed loop system without uncertainty (Eq. (20)). We can use Eq. (20) as a reference model for an adaptive controller designed as in Eqs. (4) and (6). The combined nominal and adaptive architecture is referred to as an adaptive augmented controller. Using Eqs. (4)-(6), and (15)-(21), the error dynamics can be represented as

$$
\left[\begin{array}{c}
\dot{e} \\
\dot{\tilde{\theta}}
\end{array}\right]=\left[\begin{array}{cc}
A_{m} & \lambda_{\delta} b \omega^{T} \\
-\Gamma \omega b^{T} P & 0
\end{array}\right]\left[\begin{array}{l}
e \\
\widetilde{\theta}
\end{array}\right],
$$

where $\tilde{\theta}=\theta-\theta^{*}$, and $\theta^{*}=\left[\begin{array}{llll}\theta_{\alpha}^{*} & \theta_{q}^{*} & \theta_{\delta}^{*} & \theta_{t r m}^{*}\end{array}\right]^{T}$, and where $\theta_{\alpha}^{*}=\frac{M_{\alpha}\left(1-\lambda_{\alpha}\right)+k_{\alpha} M_{\delta}\left(1-\lambda_{\delta}\right)}{\lambda_{\delta} M_{\delta}}$, $\theta_{q}^{*}=\frac{M_{q}\left(1-\lambda_{q}\right)+k_{q} M_{\delta}\left(1-\lambda_{\delta}\right)}{\lambda_{\delta} M_{\delta}}, \theta_{\delta}^{*}=\frac{k_{\delta}\left(1-\lambda_{\delta}\right)}{\lambda_{\delta}}$, and $\theta_{t r m}^{*}=-d_{t r m}$. A block diagram of the adaptive augmented system is shown in Fig. 1.

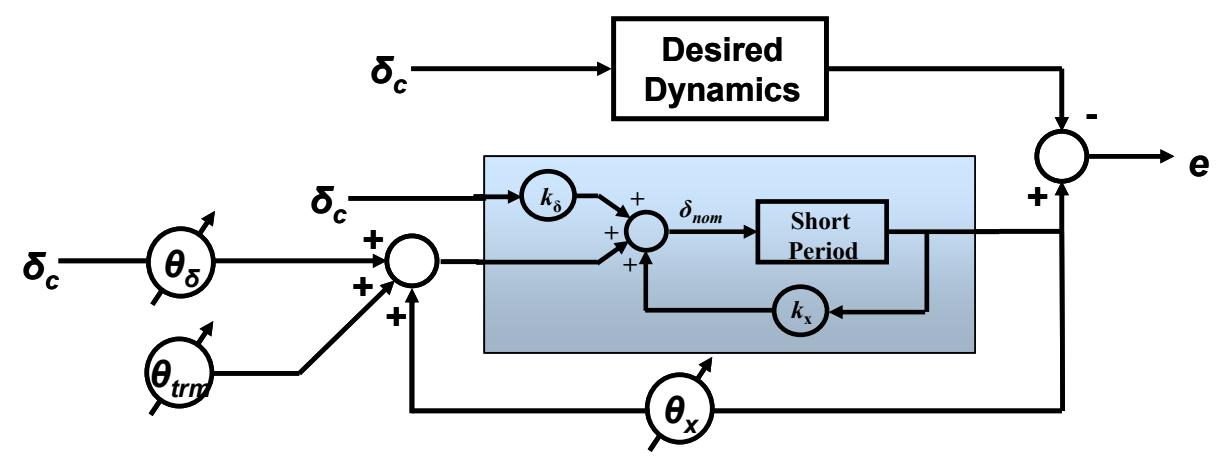

Figure 1. A block diagram showing the augmented adaptive system for the short period dynamics with multiple parameter uncertainties is shown.

The RLAS can be seen directly from Eqs. (14) and (22) to be

$$
\left[\begin{array}{c}
\dot{e}_{a} \\
\dot{\tilde{\delta}}_{a}
\end{array}\right]=\left[\begin{array}{cc}
A_{m}+\lambda_{\delta} b \theta_{x_{c}}^{T} & \lambda_{\delta} b \\
-\gamma b^{T} P & 0
\end{array}\right]\left[\begin{array}{c}
e_{a} \\
\widetilde{\delta}_{a}
\end{array}\right],
$$

where $\gamma=\omega_{m_{c}}^{T} \Gamma \omega_{m_{c}}$, and $\omega_{m_{c}}=\left[\begin{array}{lll}-\left(A_{m}^{-1} b_{m} \delta_{c}\right)^{T} & \delta_{c} & 1\end{array}\right]^{T}$.

\section{RLAS Design/Verification Procedure}

The design procedure described in section IIIB was carried out using the error dynamics in Eq. (24) and the RLAS in Eq, (23). The RLAS was tuned to produce a response within the military specification for the short period mode frequency and damping ratio (MIL-F-8785C). ${ }^{26}$ This specification requires that, for Category A flight phase (requiring rapid maneuvering) and Level 1 flying qualities (qualities adequate for the flight phase) the allowable ranges for damping ratio, $\zeta$, and natural frequency, $\omega_{n}$, are

$$
0.35 \leq \zeta \leq 1.35 \text { and } 0.653 \mathrm{rad} / s \leq \omega_{n} \leq 8.39 \mathrm{rad} / \mathrm{s}
$$

The design procedure resulted in $\Gamma=\operatorname{diag}\left(\left[\begin{array}{lllll}100 & 100 & 100 & 1\end{array}\right]\right)$ and $Q=\operatorname{diag}\left(\left[\begin{array}{ll}2 & 1\end{array}\right]\right)$ which give the damping ratio $\zeta=$ 0.518 , the natural frequency $\omega_{n}=4.95 \mathrm{rad} / \mathrm{s}$, and the $1^{\text {st }}$ order time constant $\tau=3.57 \mathrm{~s}$.

A severe failure was simulated by setting, the uncertainties to be $\lambda_{\alpha}=-1, \lambda_{q}=.9, \lambda_{\delta}=.7$, and $d_{t r m}=.1$. In Figs. 2a and $\mathrm{b}$ the responses to a random amplitude square wave input of the adaptive system and the nominal system are shown for this failure. It can be seen that the nominal system becomes unstable whereas the adaptive system is 
stable and tracks the reference model asymptotically. The response of the adaptive system clearly provides suitable flying qualities using the RLAS design methodology.

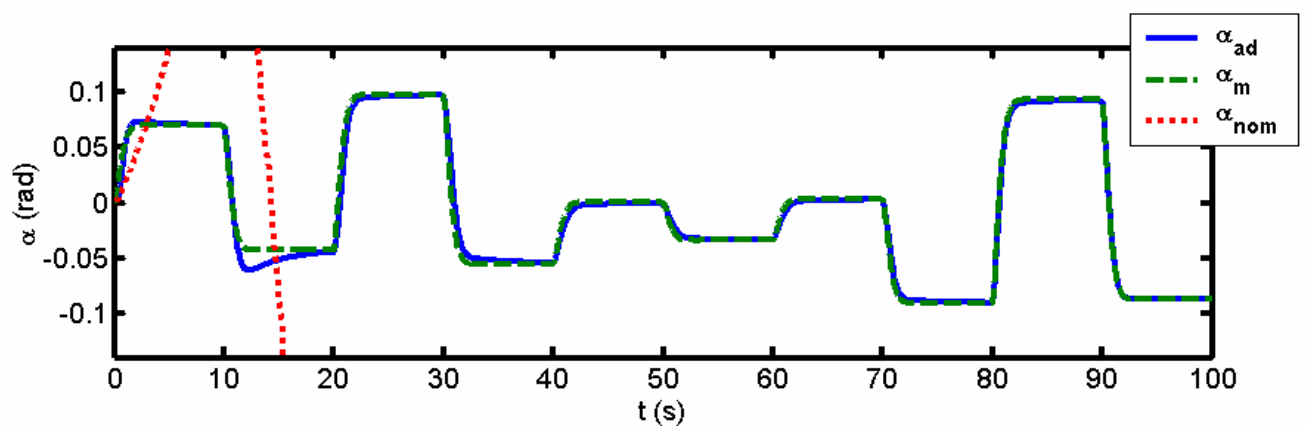

2a.

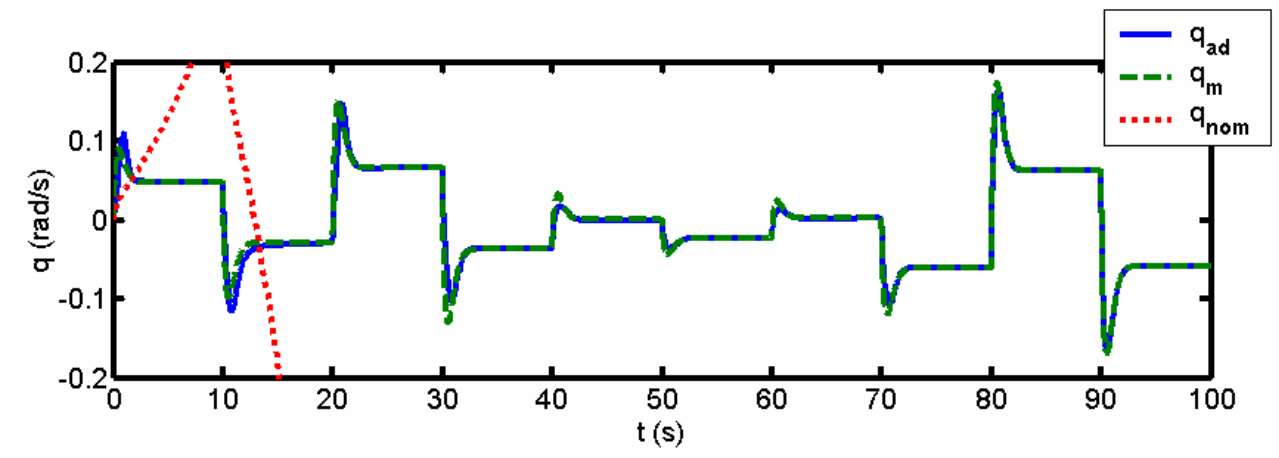

2b.

Figure 2. The $\alpha$ and $q$ trajectories of the adaptive augmented system are shown with uncertainties $\lambda_{a}=-1, \lambda_{q}=.9, \lambda_{\delta}=.7$, and $d_{t r m}=.1$. The RLAS was used to design a suitable adaptive response to a random amplitude square-wave input. The trajectories of the reference model and the nominal system are shown for comparison. Note that the nominal system is unstable.

The verification of the design was carried out according to the procedure in section IIIC. In Figs. 3a-b, and Figs. $3 \mathrm{c}-\mathrm{d}$, the trajectories of $\alpha$ and $q$ are shown for $t=0 \mathrm{~s}$ to $t=5 \mathrm{~s}$, and for $t=80 \mathrm{~s}$ to $t=85 \mathrm{~s}$ respectively, for both the adaptive system and the RLAS for the failure described previously. These figures show that the adaptive system converges to the RLAS and hence meets the specification (Eq. (24)). Even during initial transients (Figs. 3a and b) the oscillatory characteristics of the adaptive system are well approximated by the RLAS. 


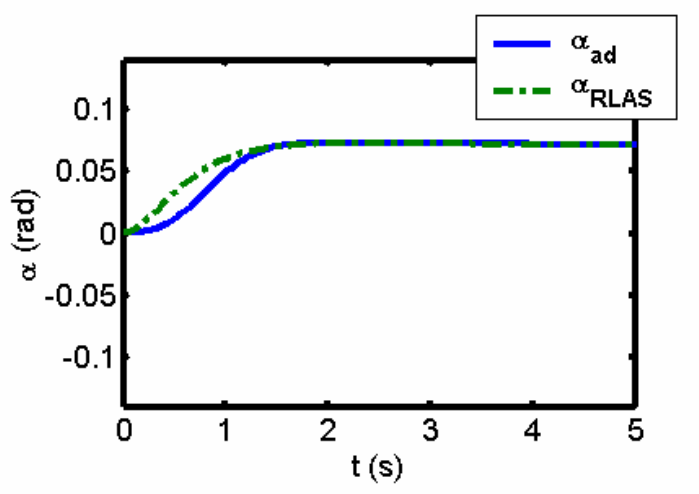

$3 a$.

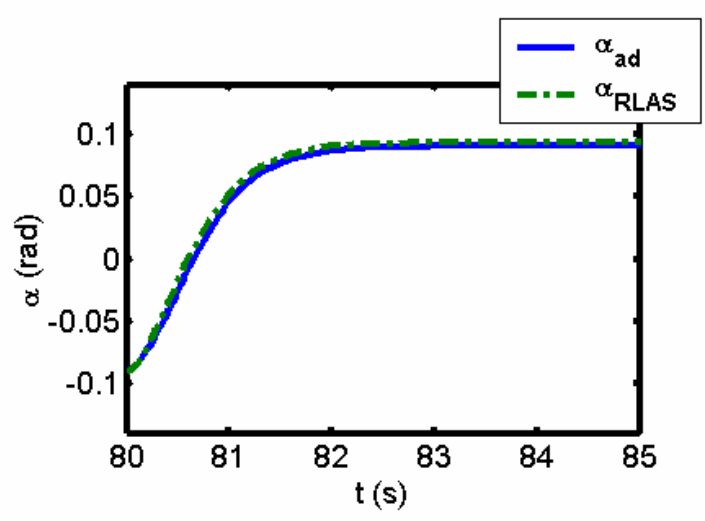

3c.

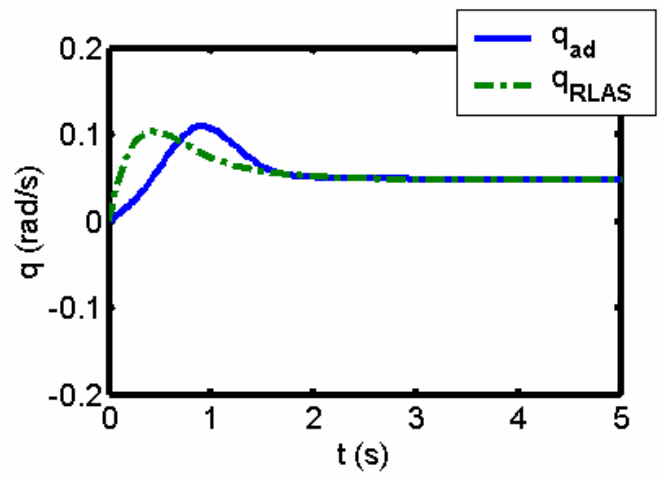

3b.

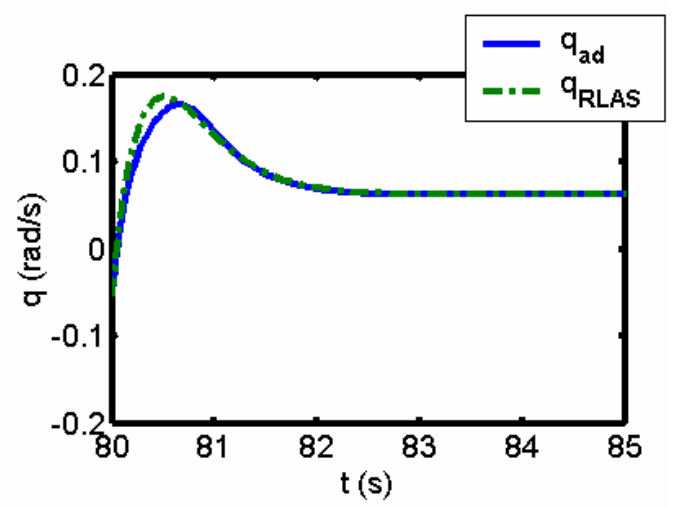

3d.

Figure 3. Close-ups of the $\alpha$ and $q$ trajectories from Fig. 2 are shown here from $t=0 \mathrm{~s}$ to $t=5 \mathrm{~s}$ (Figs. 3a and 3b), and $t=80 \mathrm{~s}$ to $t=85 \mathrm{~s}$ (Figs. 3c and 3d). The nonlinear adaptive system tracks the RLAS closely. The RLAS was designed to have one oscillatory mode with $\zeta \approx .5$ and $\omega_{n} \approx 5$, and one $1^{\text {st }}$ order mode with $\tau \approx 3.6$ s for a response well within military flying qualities specifications.

\section{Application to Nonlinear, Six Degree of Freedom Aircraft Dynamics}

A more realistic application is considered in this section, in which the design and verification techniques are applied to a full-nonlinear 6-DoF aircraft simulation. An LQ nominal controller with integral action is designed to control the short period motion of the aircraft using the uncertain, linear short period approximation in Eq. (17). The adaptive loop is closed around the closed loop nominal system to produce the adaptive augmented architecture as described in the previous section. Simulation results are presented to verify the design and demonstrate the usefulness of the RLAS.

\section{A. Model and Nominal Controller Development}

A nonlinear 6-DoF simulation of a large transport aircraft was implemented according to accepted aircraft simulation practices. ${ }^{24}$ The aircraft was trimmed with wings level to ensure that the longitudinal and lateral dynamics were decoupled. The controllers (both the LQ nominal controller and the outer loop adaptive controller) were designed using the short period approximation as a plant model to represent the nonlinear aircraft dynamics. Care was taken to ensure that the short period approximation was valid over the trajectory of interest. The nonlinear aircraft was linearized to produce the longitudinal dynamics. Then the short period approximation was used to derive the second order short period model of Eq. (17) from the fourth order longitudinal model. The short period 
dynamics in Eq. (17) serve as the plant model for the LQ nominal controller and the adaptive controller to be used with the full nonlinear aircraft.

The LQ control design technique ${ }^{25}$ is straightforwardly applied using a proportional-integral controller architecture to give zero steady state error to a step input command. The uncertain dynamics in (15) are expanded to include an integrator to give

$$
\left[\begin{array}{c}
\dot{\alpha} \\
\dot{q} \\
\dot{e}_{I}
\end{array}\right]=\left[\begin{array}{ccc}
-L_{\alpha} & -L_{q} & 0 \\
\lambda_{\alpha} M_{\alpha} & \lambda_{q} M_{q} & 0 \\
1 & 0 & 0
\end{array}\right]\left[\begin{array}{c}
\alpha \\
q \\
e_{I}
\end{array}\right]+\lambda_{\delta}\left[\begin{array}{c}
0 \\
M_{\delta} \\
0
\end{array}\right]\left(\delta+d_{t r m}\right)+\left[\begin{array}{c}
0 \\
0 \\
-1
\end{array}\right] \delta_{c},
$$

where $e_{I}$ is the state of an AOA integrator and $\delta=k_{x}{ }^{T} x+\delta_{a d}$. Equation (25) can be rewritten in the compact form $\dot{x}=A x+\lambda_{\delta} b\left(\delta+d_{t r m}\right)+b_{m} \delta_{c}$, and without uncertainty the dynamics are denoted

$$
\left[\begin{array}{c}
\dot{\alpha}_{m} \\
\dot{q}_{m} \\
\dot{e}_{I m}
\end{array}\right]=\left[\begin{array}{ccc}
-L_{\alpha} & -L_{q} & 0 \\
M_{\alpha}+M_{\delta} k_{\alpha} & M_{q}+M_{\delta} k_{q} & M_{\delta} k_{e} \\
1 & 0 & 0
\end{array}\right]\left[\begin{array}{c}
\alpha_{m} \\
q_{m} \\
e_{I m}
\end{array}\right]+\left[\begin{array}{c}
0 \\
0 \\
-1
\end{array}\right] \delta_{c},
$$

or, compactly, $\dot{x}_{m}=A_{m} x+b_{m} \delta_{c}$. The feedback gain, $k_{x}$, is found as before by minimizing the cost function in Eq. (19) subject to the dynamics in Eq. (25) with no parameter uncertainty. A suitable closed loop response was found with $Q=\operatorname{diag}\left(\left[\begin{array}{lll}1 & 1 & 10\end{array}\right]\right)$ and $R=1$ to give the feedback gains $k_{x}=\left[\begin{array}{lllll}1.2996 & 0.5305 & 3.1623\end{array}\right]^{T}$. The controller described above was applied to the nonlinear aircraft. Figures $4 \mathrm{a}$ and $\mathrm{b}$ show the response of the closed loop nominal system without uncertainty (dashed line) to an elevator doublet.

\section{B. Adaptive Augmentation and RLAS}

The system in Eq. (25) is augmented with adaptation as in Eqs. (4) and (6), using the state error vector $e=[(\alpha-$ $\left.\left.\alpha_{m}\right)\left(q-q_{m}\right)\left(e_{I}-e_{I m}\right)\right]^{T}$. The resulting error dynamics can be expressed

$$
\left[\begin{array}{c}
\dot{e} \\
\dot{\tilde{\theta}}
\end{array}\right]=\left[\begin{array}{cc}
A_{m} & \lambda_{\delta} b \omega^{T} \\
-\Gamma \omega b^{T} P & 0
\end{array}\right]\left[\begin{array}{l}
e \\
\widetilde{\theta}
\end{array}\right],
$$

where $\omega$ and $\widetilde{\theta}$ are as defined previously in Eq. (5). As before, the reference model is chosen to be the closed-loop dynamics without uncertainty as in Eq. (26). The RLAS of the closed loop aircraft with optimal PI controller and adaptive augmentation can be seen directly from Eqs. (14) and (27) to be

$$
\left[\begin{array}{c}
\dot{e}_{a} \\
\dot{\vec{\delta}}_{a}
\end{array}\right]=\left[\begin{array}{cc}
\left(A_{m}+\lambda_{\delta} b \theta_{x_{c}}^{T}\right) & \lambda_{\delta} b \\
-\gamma b^{T} P & 0
\end{array}\right]\left[\begin{array}{c}
e_{a} \\
\widetilde{\delta}_{a}
\end{array}\right],
$$

where $\gamma=\omega_{m_{c}}^{T} \Gamma \omega_{m_{c}}$, and $\omega_{m_{c}}$ and $\theta_{x_{c}}$ are as defined previously.

\section{RLAS Design/Verification Procedure}

As before, the design task was to provide adaptive augmentation while still meeting the military specification for short period frequency and damping (Eq. (24)). The design procedure described in section IIIB was employed. It

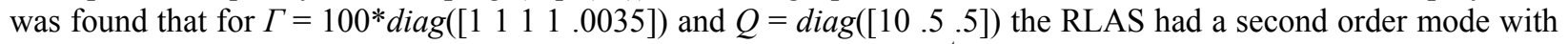
damping ratio $\zeta=0.492$, and natural frequency $\omega_{n}=1.48 \mathrm{rad} / \mathrm{s}$, and $1^{\text {st }}$ order modes with time constants $\tau_{l}=0.294 \mathrm{~s}$ and $\tau_{2}=124.8 \mathrm{~s}$. The second $1^{\text {st }}$ order mode, although it is slow, has no noticeable adverse effects on the RLAS response. 
A moderate failure and a severe failure were simulated with the nominal and adaptive controllers to compare their performance (see Figs. 4a-d). The moderate failure, parameterized by $\lambda_{\alpha}=\lambda_{q}=1, \lambda_{\delta}=.5, d_{t r m}=0.1563$, and the severe failure, parameterized by $\lambda_{\alpha}=-0.226, \lambda_{q}=-0.470, \lambda_{\delta}=0.5, d_{t r m}=0.2623$, were introduced at $t=7 \mathrm{~s}$. It was observed that the performance of the adaptive system (solid line) was similar to that of the nominal LQ controller (dotted line) for the moderate failure, but for the severe failure, the adaptive augmented system maintained stability and tracking while the system without adaptation diverged. This shows that the adaptive controller clearly outperforms the nominal controller.

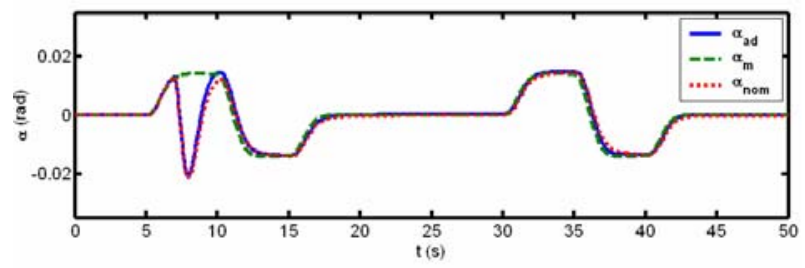

4a.

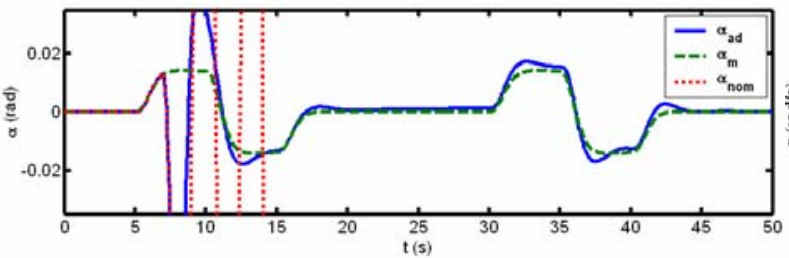

$4 c$.

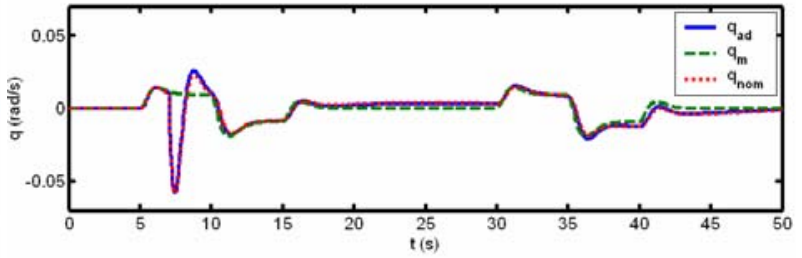

$4 b$.

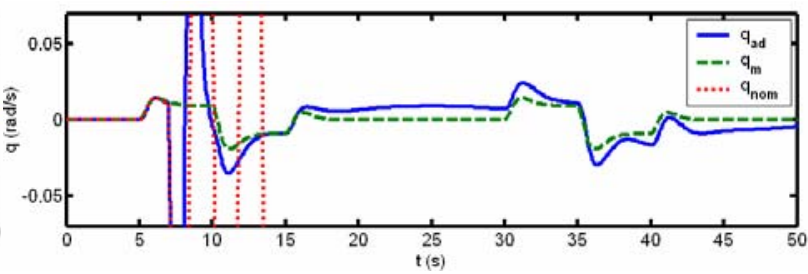

4d.

Figure 4. The response of the LQ controller (nom) and the adaptive augmented controller (ad) to two elevator doublets are shown for a moderate failure (Figs. $4 a$ and $b$ ) and a severe failure (Figs. 4c and d) occurring at $t=7 \mathrm{~s}$. The failures are parameterized by $\lambda_{\alpha}=\lambda_{q}=1, \lambda_{\delta}=.5, d_{\text {trm }}=0.1563$ and $\lambda_{\alpha}=-0.226, \lambda_{q}=-$ $0.470, \lambda_{\delta}=0.5, d_{t r m}=0.2623$ respectively. For the moderate failure, the responses of the two systems are similar, whereas for the severe failure, the system with the LQ controller becomes unstable while the adaptive augmented system maintains stability and tracking.

The severe failure in Fig. 4 was explored more carefully for the purposes of verification. Figure 5 shows the $\alpha$ and $q$ response of the adaptive system to a random amplitude square-wave input for the severe failure. After a transients of about five seconds, adequate model following is achieved for $\alpha$. For $q$, the unmodeled phugoid mode can be clearly observed in the $q$ trajectory of the nonlinear aircraft. The phugoid mode for this aircraft has a frequency of $\omega_{n}=0.122 \mathrm{rad} / \mathrm{s}$ corresponding to a cycle time of $51.5 \mathrm{~s}$, which agrees closely with the low frequency oscillations observed in Fig. 5b. The model mismatch due to the phugoid mode could be easily eliminated by using a more sophisticated longitudinal reference model, namely one including phugoid dynamics. This exercise demonstrates that even with the crudest of plant models an adaptive controller with adequate transient properties can be realized using the RLAS. 


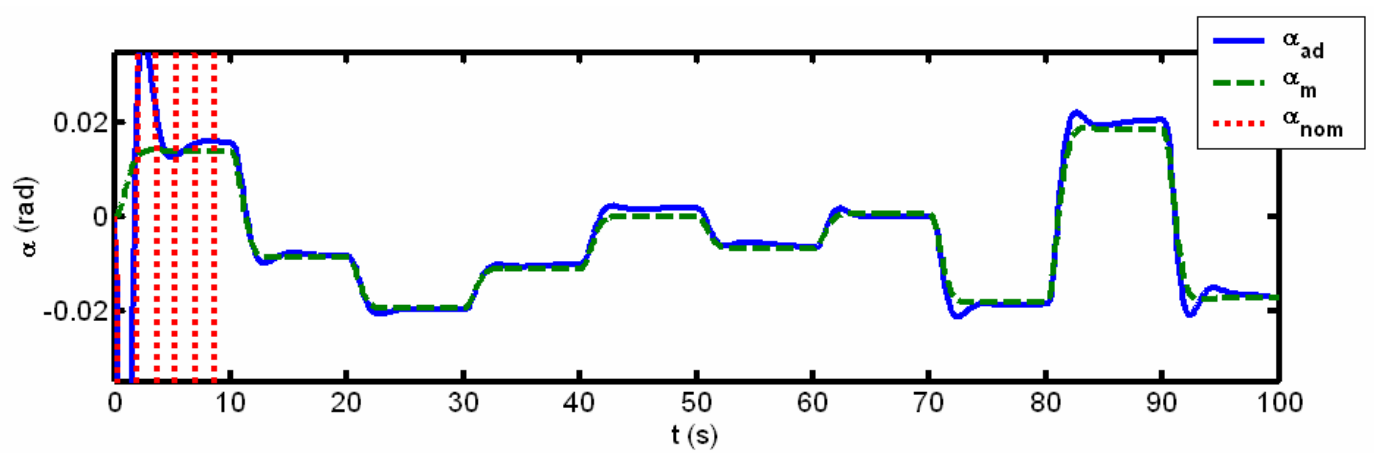

5a.

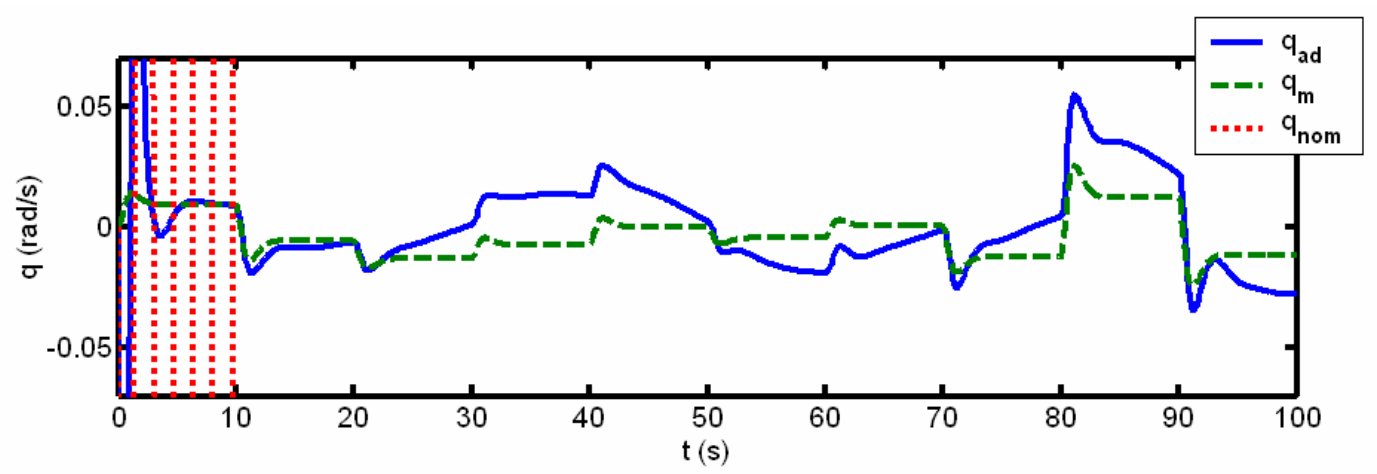

5 b.

Figure 5. The $\alpha$ and $q$ trajectories of the adaptive augmented system are shown with uncertainties parameterized by $\lambda_{\alpha}=-\mathbf{0 . 2 2 6}, \lambda_{q}=-0.470, \lambda_{\delta}=0.5$, and $d_{\text {trm }}=0.2623$. The low frequency oscillations in the $q$ trajectory are from the unmodelled phugoid mode of the nonlinear aircraft. The RLAS was used to design a suitable adaptive response to a random amplitude square-wave input. The trajectories of the reference model and the nominal system are shown for comparison. Note that the nominal system is unstable.

The verification procedure described in section IIIC was carried out for the severe failure. Figures $6 \mathrm{a}$ and $\mathrm{b}$ show in detail the first five seconds of the response in comparison to the RLAS. During the transient period, although the RLAS does not track the nonlinear system closely, its frequency of oscillation provides a good representation of the oscillations of the nonlinear aircraft. As the adaptive system evolves, the RLAS provides a more accurate representation of its trajectory (see Figs. 6c and d). Again, the offset of the nonlinear system in Fig. 6d is attributed to the unmodelled phugoid mode. It is clear that the RLAS tracks the adaptive system relatively closely even for the nonlinear aircraft model. 


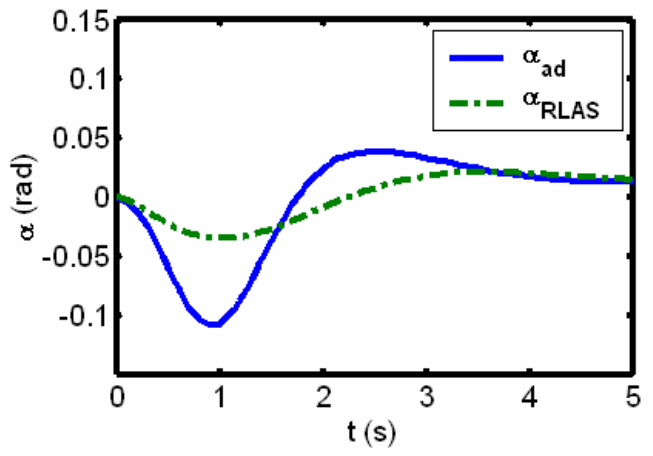

6a.

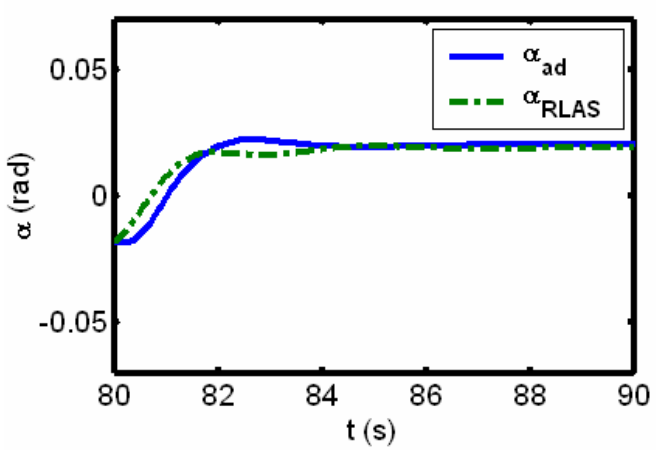

6c.

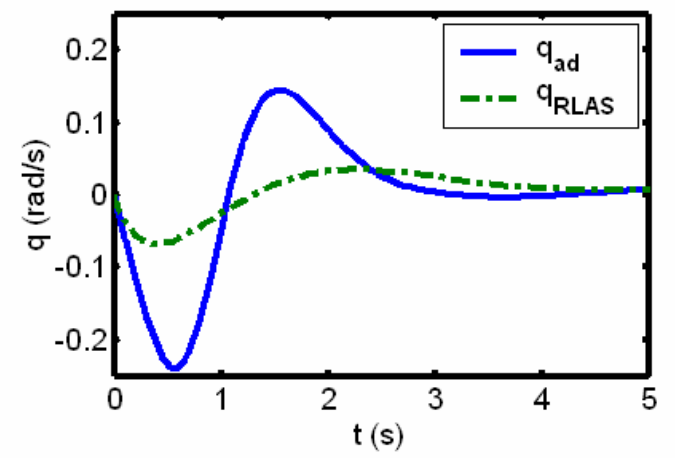

6b.

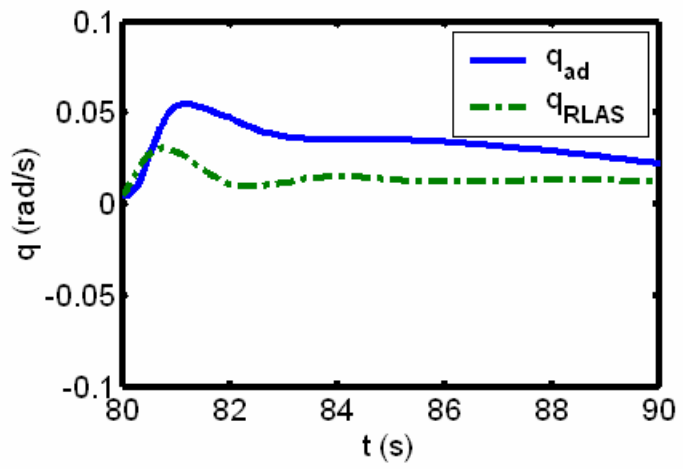

6d.

Figure 6. The $\alpha$ and $q$ trajectories from Fig. 5 are shown here in detail from $t=0 \mathrm{~s}$ to $t=5 \mathrm{~s}$ (Figs. 6a and b), and from $t=80 \mathrm{~s}$ to $t=85 \mathrm{~s}$ (Figs. 6c and d). The RLAS (dash-dot) gives a good indication of the adaptive response frequency (solid) initially, and tracks the adaptive $\alpha$ response well after initial transients. The offset in the $q$ trajectory is from the unmodelled phugoid mode. The RLAS was designed to have a response well within military flying qualities specifications.

It should be stressed, as well, that the closed loop trajectory is that of a full nonlinear aircraft model with an LQ controller and an adaptive controller. Thus the system is highly nonlinear, yet its qualities of oscillation can be inferred from the simple linear RLAS to a practically useful degree. Figure 6 verifies that the adaptive system response is within the military flying qualities specification (Eq. (24)) as expected from the RLAS design procedure.

\section{Summary}

In this paper we have sought to introduce a new tool for designing and verifying the oscillatory properties of adaptive systems. The RLAS was formulated to provide a compact linear approximation to nonlinear adaptive systems. General design and verification techniques using the RLAS were then described. An adaptive augmented system was design using the RLAS techniques for a linear model of short period dynamics. The adaptive system was verified to have properties that meet military specifications. The same design techniques were then applied to a nonlinear 6-DOF aircraft simulation. Again, it was demonstrated that an adaptive controller can be tuned to meet military specifications using the RLAS.

Although not explicitly perused here, the RLAS can also be used as a tool to explore the effects of failures on the adaptive system dynamics. Uncertainties in the adaptive dynamics translate directly to uncertainties in the RLAS. The effect of these uncertainties on the RLAS can be straightforwardly investigated using the common linear systems techniques, such as the root locus method, Bode plots, or Nyquist plots. Again, relying on the asymptotic convergence of the RLAS to the adaptive system, we can infer the effects of failures on the adaptive system response. The procedure described in this paper can be simply modified for this purpose.

Several avenues of research remain ongoing. An analytically justified means of estimating the asymptotic gain error $\theta_{x c}$ is currently being investigated. Also, multi-input embodiments of the RLAS should be explored in 
conjunction with multivariable design techniques. One potentially interesting extension of the RLAS design methodology is to use an optimal technique to select adaptive parameters $\Gamma$ and $Q$, thereby fusing the benefits of optimal and adaptive control. Modifying current control design techniques to suit adaptive controllers will likely aid the transition of adaptive control technologies into aircraft and other safety critical applications.

\section{Acknowledgments}

This research was supported by the Boeing Strategic University Initiative.

\section{References}

${ }^{1}$ Narendra, K. S., and Annaswamy, A. M., Stable Adaptive Systems, Prentice-Hall, Englewood Cliffs, NJ, 1989.

${ }^{2}$ Slotine, J. J. E., and Li, W., Applied Nonlinear Control, Prentice Hall, Englewood Cliffs, NJ, 1991.

${ }^{3}$ Wohletz, J. M., Paduano, J. D., Annaswamy, A. M., "Retrofit Systems for Reconfiguration in Civil Aviation," Proc. AIAA Guidance, Navigation, and Control Conf., Portland, OR, Aug. 1999, pp. 995-1005.

${ }^{4}$ Bodson, M., and Groszkiewicz, J. E., "Multivariable Adaptive Algorithms for Reconfigurable Flight Control," IEEE Transactions on Control Systems Technology, Vol. 5, No. 2, Mar. 1997, pp. 217 - 229.

${ }^{5}$ Kim, K. S., Lee, K. J., and Kim, Y., "Model Following Reconfigurable Flight Control System Design Using Direct Adaptive Scheme," Proc. AIAA Guidance, Navigation, and Control Conf., Monterey CA, Aug. 5-8, 2002.

${ }^{6}$ Tao, G., Joshi, S. M., and Ma, X., "Adaptive State Feedback and Tracking Control of Systems with Actuator Failures," IEEE Transaction on Automatic Control, Vol. 46, No. 1, Jan. 2001, pp. 78-95.

${ }^{7}$ Boskovic, D. J., and Mehra, R. K., "Multiple Model Adaptive Flight Control Scheme for Accommodation of Actuator Failures," AIAA Journal of Guidance, Control, and Dynamics, Vol. 25, No. 4, 2002, pp. 712-724.

${ }^{8}$ Calise, A. J., Lee, S., and Sharma, M., "Development of a Reconfigurable Flight Control Law for Tailless Aircraft," AIAA Journal of Guidance, Control, and Dynamics, Vol. 24, No. 5, Sept.-Oct. 2001.

${ }^{9}$ Henderson, D. K., and Lavretsky, E. Y., "Closed-Loop Model Following and Control Allocation for Transport Aircraft Using an Ordered Neural Network-Based Approach," Proc. AIAA Guidance, Navigation, and Control Conf., Portland, OR, Aug. 9-11, 1999.

${ }^{10}$ Karason, S. P., and Annaswamy, A. M., "Adaptive Control in the Presence of Input Constraints," IEEE Transactions on Automatic Control, Vol. 39, No. 11, Nov. 1994, pp. 2325-2330.

${ }^{11}$ Schwager, M., and Annaswamy, A. M., "Multi-input Training Signal Hedging for a States Accessible Aircraft System," Active-Adaptive Control Laboratory, Technical Report 0403, Department of Mechanical Engineering, M.I.T., Sept. 2004.

${ }^{12}$ Lavretsky, E., and Hovakimyan, N., "Positive $\mu$-modification for stable adaptation in a class of nonlinear systems with actuator constraints," Proc. American Control Conf. Boston, MA, July 1-4, 2004

${ }^{13}$ Schwager, M., and Annaswamy, A. M., "Adaptation-Based Reconfiguration in the Presence of Actuator Failures and Saturation," Submitted to the AIAA Guidance Navigation and Control Conf., San Francisco, CA, Aug. 15-18, 2005.

${ }^{14}$ Buus, H., McLees, R., Orgun, M., Pasztor, E., and Schultz, L., "777 Fight Controls Validation Process," IEEE Trans. on Aerospace and Electronic Systems, Vol. 33, No. 2, 1997, pp.656-66.

${ }^{15}$ Duke, E. L., "V\&V of Fligth and Mission-Critical Software," IEEE Software Magazine, May 1989, pp.39-45.

${ }^{16}$ Renfrow, J., Leibler, S., Denham, J., "F-14 Flight Control Law Design, Verification, and Validation Using Computer Aided Engineering Tools," Naval Air Warfare Center, Aircraft Div., Rept. A762492, Patuxent River, MD, Feb. 1995.

${ }^{17}$ Tomlin, C. J., Mitchell, I., Bayen, A. M., and Oishi, M., "Computational Techniques for the Verification of Hybrid Systems," Proc. IEEE Conf. on Decision and Control., Vol. 91, No. 7, July 2003.

${ }^{18}$ Cukic, B., "The Need for Verification and Validation Techniques for Adaptive Control Systems," Proc. International Symposium on Autonomous Decentralized Systems, Dallas, TX, Mar. 2001, pp. 297-8.

${ }^{19}$ Cortellessa, V., Cukic, B., Del Gobbo, D., Mili, A., Napolitano, M., Shereshevsky, M., Sandhu, H., "Certifying Adaptive Flight Control Software," Proc. International Software Assurance Certification Conference, Reston, VA, Sept. 25-26, 2000.

${ }^{20}$ James, J., and Barton, D., "A Framework for Verification and Validation of Integrated and Adaptive Control Systems," Proc. IEEE Int. Symp. on Computer-Aided Control Systems Design, Anchorage, AK, Sept. 2000.

${ }^{21}$ Hull, J., and Ward, D., "Verification and Validation of Neural Networks for Safety-Critical Applications," Proc. American Control Conf., Anchorage, AK, May 8-10, 2002.

${ }^{22}$ Liu, Y., Gururajan, S., Cukic, B., Menzies, T., Napolitano, M., "Validating an Online Adaptive System Using SVDD," $15^{\text {th }}$ IEEE Int. Conf. on Tools with Artificial Intelligence, Sacramento, CA, Nov. 3-5, 2003.

${ }^{23}$ Schwager, M., and Annaswamy, A. M., "Parameter Error Convergence in Adaptive Systems with Constant Inputs," ActiveAdaptive Control Laboratory, Technical Report 0501, Department of Mechanical Engineering, M.I.T., Jan. 2005.

${ }^{24}$ Stevens, B. L., and Lewis, F. L., Aircraft Simulation and Control, $2^{\text {nd }}$ ed., John Wiley \& Sons, Inc, Hoboken, NJ, 2003.

${ }^{25}$ MATLAB Control System Toolbox Help, The Math Works, Ver. 6.0.0.88, Release 12, Sept. 2000.

${ }^{26}$ MIL-F-8785C. "U.S. Dept. of Defense Military Specification: Flying Qualities of Piloted Airplanes.” Nov. 5, 1980. 